\title{
Prediction of Harvested Energy for Wireless Sensor Node
}

\author{
Strahinja Jankovic*, Lazar Saranovac \\ School of Electrical Engineering, University of Belgrade, \\ Bulevar kralja Aleksandra 73, 11020 Belgrade, Serbia \\ jankovics@etf.bg.ac.rs
}

\begin{abstract}
Energy harvesting wireless sensor nodes are interesting for the Internet of Things, since they can provide continuous operation by adapting workload not only to the current energy reserves, but to the amount of energy that can be harvested in the future also. We present a multistage day ahead hourly solar energy prediction algorithm. The predictor uses cloud cover and precipitation probability predictions from weather forecast obtained once per day for 24 hours in advance. To compensate for short-term weather changes until the next weather forecast data is obtained, forecast errors of humidity and atmospheric pressure are fed to the fuzzy logic filter. The filter adjusts predictions of cloud cover and precipitation probability, which are applied to the clear-sky radiation model in order to obtain prediction of solar energy. The prediction of solar energy is additionally corrected based on the energy prediction error in the preceding time slot. The results show that the proposed predictor outperforms the stateof-the-art predictors in terms of prediction error. Proposed predictor and state-of-the-art predictors were also evaluated using a simulated wireless sensor node with the simple energy management algorithm, where the proposed predictor was the most efficient at maintaining energy neutrality.
\end{abstract}

Index Terms-Energy harvesting; Energy management; Fuzzy logic; Solar energy; Wireless sensor network.

\section{INTRODUCTION}

Modern pervasive environment integrates multitude of sensors, which provide data that can be used to improve the quality of life. Deployed sensors have found their use in various aspects of everyday life like monitoring of air quality, weather conditions, agriculture production, medical devices, as well as various safety applications like monitoring of bridge stability, earthquake, and volcanic activities [1]-[3]. Sensors can be placed in static locations or attached to mobile platforms [4].

Sensors are interfaced with sensor nodes that have certain processing capability, which acquire and/or process data from the sensors and transmit the data for further use via wired or wireless network connection. Multiple sensor nodes can be organized into sensor networks. Internet of Things [5] enables integration of sensor networks and nodes and management of acquired data. The advance of big data

Manuscript received 11 July, 2019; accepted 15 January, 2020.

This work was supported by the Serbian Ministry of Education, Science and Technological Development under grant (No. TR32043). algorithms [6] that can be used to process this amount of data enable development of new context, in which the data can be used, thus making new applications possible.

Sensor nodes that are deployed outside or in remote locations are usually battery powered. Thus, reduced power/energy consumption is one of the requirements when designing such sensor nodes. Besides design-time optimization, different energy management algorithms exist on the node-level and/or network-level, which can be used at run-time to prolong node operation before the battery is, discharged [7]. Node-level energy management algorithms conserve energy either by reducing performance like dynamic voltage and frequency scaling, duty cycling, adaptive sampling or disabling components when they are not in use like power/clock gating. Network-level energy management algorithms are based on using energy efficient routing and MAC protocols, clustering or data management, including data compression and aggregation. However, regardless of how effective energy management algorithm is, the battery will be depleted eventually. That is a problem, since battery replacement can be hard or even impossible depending on the number of deployed nodes or location, where nodes are deployed.

Energy harvesting is a promising solution to the finite battery capacity problem. Various environmental energy resources are available like solar, wind, vibration, and radio frequency (RF). These energy resources can be categorized based on their controllability and predictability [8]. For instance, solar energy cannot be controlled, but it is predictable based on its diurnal cycle.

With energy harvesting, the application optimization focus is turned towards uninterrupted operation and optimal allocation of available energy defined as the concept of energy neutrality [9]. The goal of energy neutrality is to adapt node operation, so the battery never gets depleted while maximizing the amount of work that is performed. In order to make energy neutrality possible, the prediction of the future available energy is needed. Besides prediction, the design of the energy allocation algorithm is also important, which is not the focus of this paper.

There are many proposed solutions for predicting future available energy. They can be divided into Past Predicts the Future (PPF) and weather forecast based approaches. In the following analysis, we will focus only on the solar energy prediction approaches. 


\section{A. PPF Techniques}

PPF techniques rely on the solar energy diurnal cycle. Days are divided into time slots with length of 30 to 60 minutes. Future available solar energy is calculated based on the energy measured in the preceding days and/or the preceding time slots.

The authors in [9] have presented the Exponentially Weighted Moving Average (EWMA) algorithm that uses the diurnal nature of solar radiation and calculates prediction for the same time slot in the following day using

$$
\hat{E}_{d+1}^{t}=\alpha \hat{E}_{d}^{t}+(1-\alpha) E_{d}^{t}
$$

where $E_{d}^{t}$ and $\hat{E}_{d}^{t}$ are measured and predicted energy, respectively, in time slot $t$ on day $d$, and $\alpha<1$ is a weight factor. EWMA gives good results if weather conditions between consecutive days remain the same, but prediction errors increase when weather conditions change, especially between days.

Weather-Conditioned Moving Average (WCMA) [10] uses exponential smoothing in a different manner. Instead of predicting available energy in the same time slot for the following day, prediction is made for the following time slot. The downside of WCMA is that it can be used just for shortterm prediction, since it can provide prediction only for the following time slot.

Profile energy prediction model (Pro-Energy) [11] further extends the WCMA idea by searching the list of $D$ stored measured solar energy profiles for the most similar to the current day and using it for further prediction. Similarity is defined as having lesser Mean Absolute Error (MAE) between current day measurements and stored measured energy profile in the preceding $K$ time slots. The window is limited to the preceding $K$ time slots, so prediction can adapt to changing weather conditions during the day. Since storage of the measured energy profiles is limited, new profiles are obtained by combining $P$ most similar profiles into the weighted profile $W P$ by using

$$
W P^{t+1}=\frac{1}{P-1} \sum_{j=1}^{P} \omega_{j} \times E_{d-j}^{t+1},
$$

where

$$
\omega_{j}=1-\frac{\operatorname{MAE}_{K}\left(E_{d-j}, E_{d}\right)}{\sum_{j=1}^{P} M A E_{K}\left(E_{d-j}, E_{d}\right)},
$$

and

$$
M A E_{K}\left(E_{d-j}, E_{d}\right)=\sum_{i=t-K}^{t} \frac{1}{K}\left|E_{d-j}^{i}-E_{d}^{i}\right| .
$$

The prediction for the following time slot is calculated as

$$
\hat{E}_{d}^{t+1}=\alpha E_{d}^{t}+(1-\alpha) W P^{t+1} .
$$

Pro-Energy yields better results for short-term predictions compared to EWMA and WCMA [11].
The authors in [12] have presented an improved version of Pro-Energy (IPro-Energy), which introduces a "smarting factor" $S$ to compensate for interday weather changes. The "smarting factor" is calculated as

$$
S=r \times\left(\frac{E_{d}^{t}-E_{d}^{t-1}}{\left(E_{d}^{t}+E_{d}^{t-1}\right) / 2}\right) \times E_{d}^{t-1},
$$

where $r$ is a factor that determines how much abrupt change in weather conditions between preceding and current time slot impact prediction. The prediction for the following time slot is calculated as

$$
\hat{E}_{d}^{t+1}=W_{f} E_{d}^{t}+\left(1-W_{f}\right) W P^{t+1}+S
$$

where $W_{f}$ is a weight factor similar to $\alpha$ in (5).

Variations of WCMA and Pro-Energy, which use variable time slot lengths, have been presented in [13], [14]. The use of variable time slot lengths enables lower predictor memory footprint compared to fixed time slot lengths, since information regarding only characteristic slots is stored However, this approach is applicable only to PPF techniques, not the weather forecast based technique we are presenting.

\section{B. Weather Forecast Techniques}

Weather forecast data are usually available on an hourly basis for at least 24 hours ahead, so time slots for weather forecast based techniques are set to 60-minute periods.

Cloud cover information from weather forecast data has previously been used to predict solar irradiance and future available solar energy [15]-[17]. It is expressed in oktas or percentages, where higher values indicate more clouds in the sky [18]. In order to obtain expected solar irradiance when using cloud cover data, the cloudless sky solar irradiance data are needed.

The authors in [15] have shown that weather forecast prediction can give better results compared to PPF techniques for medium and long-term predictions. They have used the linear relationship between solar irradiance and solar panel energy output to calculate the expected solar energy. The cloudless sky solar energy is obtained using the quadratic function

$$
E_{C S}^{t}=a \times(t+b)^{2}+c
$$

where $a, b$, and $c$ are parameters that have been experimentally determined for each month of the year, and $t$ is time of the day.

The expected solar energy is calculated as

$$
\hat{E}^{t}=\left(1-C C^{t}\right) E_{C S}^{t}
$$

where $C C^{t}$ is the cloud cover data for time slot $t$.

The concept from [15] has been further explored in [16], [17], where prediction output is used to schedule tasks. The results have shown that higher number of tasks are executed using this approach compared to PPF approaches. 
In our previous paper [19], we have used Photovoltaic Geographical Information System (PVGIS) [20] to obtain cloudless sky solar irradiance data. PVGIS provides monthly, daily, and hourly solar irradiance data. The expected solar irradiance has been calculated as

$$
\widetilde{G}^{t}=\left(1-C C^{t}\right) G_{b}^{t}+G_{d}^{t},
$$

where $G_{b}^{t}$ is direct (beam) solar irradiance and $G_{d}^{t}$ is diffuse solar irradiance obtained from PVGIS. The expected solar energy $\widetilde{E}^{t}$ has been calculated based on expected solar irradiance for time slot $t$ and solar panel characteristics. The prediction of solar energy for the following time slot is obtained by using

$$
\hat{E}^{t}=(1+\alpha) \widetilde{E}^{t}
$$

where

$$
\alpha=\frac{E^{t-1}-\tilde{E}^{t-1}}{\tilde{E}^{t-1}}, \alpha \in[-0.3,0.05] .
$$

The $\alpha$ factor is used to correct prediction based on forecast error in the preceding time slot. However, the correction in (12) is bound. Therefore, it underestimates available energy. In addition, the downside of PVGIS is that it is based on the measured data, which already include the effect of cloud cover.

We present a multistage day ahead solar energy prediction algorithm that uses clear-sky solar radiation model and applies cloud cover and precipitation prediction data from 24 hours ahead weather forecast to predict available solar energy. Since weather forecast data are obtained once per day, fuzzy logic filter with humidity and atmospheric pressure forecast error data as inputs are used to correct cloud cover and precipitation prediction data. Additionally, correction based on prediction error in preceding time slot is used during the day to update the expected energy in order to compensate for forecast errors.

Compared to our previous work, the following improvements are made:

1. Clear-sky radiation model is used instead of PVGIS [20] data. PVGIS data are based on ground measurements, so the effects of cloud cover are included in the data and expected solar energy is on the lower bound. Unlike PVGIS, the clear-sky radiation model provides maximum possible irradiance data.

2. In addition to cloud cover, the precipitation prediction data from weather forecast is used. The precipitation affects the diffuse component of irradiance [21].

3. Fuzzy logic filter is used for adjusting cloud cover and precipitation prediction data based on forecast error of humidity and atmospheric pressure. This step enables correction of forecast data for weather changes during the night.

Proposed predictor has been compared to Pro-Energy and IPro-Energy, state-of-the-art PPF predictors, and our previous work [19] in terms of prediction error, and it gives better prediction results. Additionally, all predictors have been tested on a simulated wireless sensor node with a simple energy management algorithm in order to evaluate predictors' ability to maintain energy neutrality. The results show that proposed predictor is the most efficient in maintaining energy neutrality.

The rest of the paper is organized as follows. In Section II, the clear-sky solar radiation model is explained and the relation between extraterrestrial solar irradiance, cloud cover, and precipitation probability for calculation of ground global irradiance is introduced. The details of proposed predictor are presented in Section III. In Section IV, the model of energy harvesting wireless sensor node is given. Results and discussion are introduced in Section V. The conclusions are presented in Section VI.

\section{SOLAR IRRADIANCE AND ENERGY}

In order to calculate available solar energy, information about global, direct and diffuse solar irradiance is needed. We are using the extraterrestrial solar radiation model in order to calculate the cloudless sky solar irradiance for the given day and hour. In the following paragraphs, it is considered that solar panel is placed tangentially to the Earth's surface. Detailed calculations can be found in [22], if panel is tilted.

Extraterrestrial irradiance represents solar energy that falls on a horizontal surface outside the atmosphere. It depends on a distance between Earth and Sun, and it can be calculated as follows

$$
G_{o}=G_{S C}\left(1+0.0033 \cos \frac{360^{\circ} \times d_{n}}{365.25}\right) \cos \theta_{z},
$$

where $G_{S C}=1367 \mathrm{~W} / \mathrm{m}^{2}$ is solar constant, $d_{n}$ is day of the year, and $\theta_{z}$ is zenith angle. The value of zenith angle can be calculated from

$$
\theta_{z}=\cos ^{-1}(\cos \phi \cos \delta \cos \omega+\sin \phi \sin \delta),
$$

where $\phi$ is panel position latitude, $\delta$ is declination, and $\omega$ is the hour angle, which depends on the panel position longitude. The declination $\delta$ is calculated as

$$
\begin{gathered}
\delta=(0.006918-0.399912 \cos \Gamma+0.070257 \sin \Gamma- \\
-0.006758 \cos 2 \Gamma+0.000907 \sin 2 \Gamma- \\
-0.002697 \cos 3 \Gamma+0.00148 \sin 3 \Gamma)(180 / \pi),
\end{gathered}
$$

where $\Gamma$ is the day angle calculated as

$$
\Gamma=2 \pi \frac{d_{n}-1}{365} .
$$

The hour angle $\omega$ is calculated as

$$
\omega=15^{\circ} \times(12-S T)
$$

where $S T$ is solar time. The solar time $S T$ is obtained using 


$$
S T=t+\frac{E_{t}+4\left(\text { lon }-15^{\circ} \times T Z\right)}{60},
$$

where $E_{t}$ is equation of time, lon is panel position longitude, and $T Z$ is time zone. The equation of time $E_{t}$ is calculated as follows

$$
\begin{aligned}
E_{t}= & (0.000075+0.001868 \cos \Gamma-0.032077 \sin \Gamma- \\
& -0.014615 \cos 2 \Gamma-0.04089 \sin 2 \Gamma) \times 229.18 .
\end{aligned}
$$

Once extraterrestrial solar irradiance has been calculated, we then apply the clear-sky solar radiation model.

From Hottel [23], clear-sky direct irradiance can be calculated from extraterrestrial irradiance and zenith angle as

$$
G_{b}=G_{o}\left(a_{0}+a_{1} e^{-\frac{k}{\cos \theta_{z}}}\right)
$$

where $a_{0}, a_{1}$, and $k$ are parameters that depend on climate type. These parameters are calculated from (21)-(23):

$$
\begin{aligned}
& a_{0}=r_{0}\left(0.4237-0.00821(6-A)^{2}\right), \\
& a_{1}=r_{1}\left(0.5055+0.00595(6.5-A)^{2}\right), \\
& k=r_{k}\left(0.2711+0.01858(2.5-A)^{2}\right),
\end{aligned}
$$

where $A$ is altitude and $r_{0}, r_{1}$, and $r_{k}$ are correction factors for different climate types given in Table I.

TABLE I. VALUES OF CORRECTION FACTORS $r_{0}, r_{1}$, AND $r_{k}$ FOR DIFFERENT CLIMATE TYPES [23].

\begin{tabular}{|c|c|c|c|}
\hline Climate type & $\boldsymbol{r}_{\mathbf{0}}$ & $\boldsymbol{r}_{\mathbf{1}}$ & $\boldsymbol{r}_{\mathbf{k}}$ \\
\hline Tropical & 0.95 & 0.98 & 1.02 \\
\hline Midlatitude summer & 0.97 & 0.99 & 1.02 \\
\hline Subarctic summer & 0.99 & 0.99 & 1.01 \\
\hline Midlatitude winter & 1.03 & 1.01 & 1.00 \\
\hline
\end{tabular}

The diffuse irradiance is calculated according to Liu and Jordan [24] as

$$
G_{d}=0.271 G_{o}-0.294 G_{b}
$$

The clear-sky global irradiance is the sum of direct and diffuse components

$$
G=G_{b}+G_{d}
$$

It should be noted that the value of clear-sky global irradiance (25) depends only on the geographical position (latitude, longitude, and altitude) and time, so it can be precomputed and used as a lookup table, if these parameters are known.

As stated previously, the cloud cover is used as a parameter, which modulates clear-sky direct irradiance. Compared to our previous work [19], we have also included precipitation probability, since it has been shown that it can affect solar radiation reaching Earth's surface [21]. We used precipitation probability data to modulate the clear-sky diffuse irradiance $G_{d}$.

In order to obtain expected solar irradiance from weather forecast data, we apply cloud cover and precipitation probability to direct (20) and diffuse (24) components, respectively, as

$$
\widetilde{G}^{t}=\left(1-C C^{t}\right) G_{b}^{t}+\left(1-P P^{t}\right) G_{d}^{t},
$$

where $C C^{t}$ is cloud cover and $P P^{t}$ is precipitation probability for time slot $t$.

\section{PROPOSED PREDICTOR}

Proposed predictor uses the clear-sky radiation model and applies cloud cover and precipitation probability prediction obtained from weather forecast. In order to minimize data transmission, we obtain 24 hours ahead forecast once per day, at high noon, when it is expected that most energy will be available.

The downside of analyzed PPF algorithms is that they cannot predict the early morning solar energy, since there is no solar energy data during the night to compare to. The weather forecast based techniques have the advantage that weather forecast data are available at least up to 24 hours ahead. However, since forecast error increases with the length of forecast period, the actual error depends on the rate that forecast data are obtained. Additionally, cloud cover is not observable during the night, so prediction errors can be hard to detect before morning.

The effect of prediction errors on energy that is stored is most noticeable early in the morning and in the evening. Errors in the evening do not have to be catastrophic, since node operation can be adjusted during the night to compensate for error. However, errors in the morning can lead to failure in operation, since battery can get depleted. It should be noted that PPF algorithms are less susceptible to the early morning errors, since prediction for the following time slot is affected by the preceding time slot, thus creating a delay of a time slot between actual and predicted energy. Prediction errors for the weather forecast based approaches depend heavily on the quality of the used weather forecast.

In order to tackle this problem, we have introduced a fuzzy filter stage to our predictor. We use forecast errors of humidity and atmospheric pressure to modify cloud cover and precipitation probability obtained from weather forecast.

The correlation between actual cloud cover and humidity and atmospheric pressure is positive [25] and negative [26], respectively. Membership functions for fuzzified values of forecast error of humidity $\Delta H$ and atmospheric pressure $\Delta P$ have the shape shown in Fig. 1.

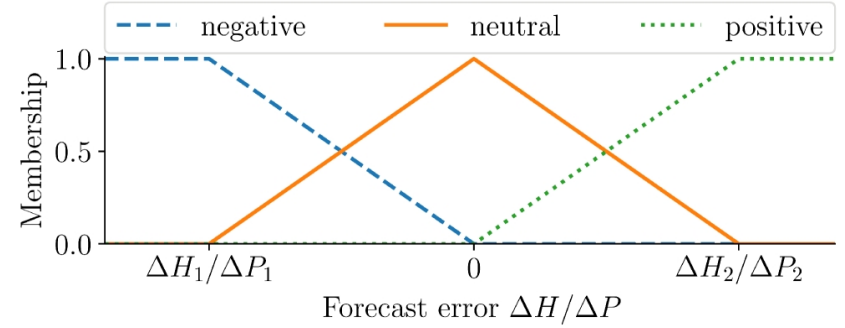

Fig. 1. Membership functions for forecast errors of humidity $(\Delta H)$ and atmospheric pressure $(\Delta P)$. 
Membership functions for correction of cloud cover $\triangle C C$ and precipitation prediction $\triangle P P$ have the shape shown in Fig. 2.

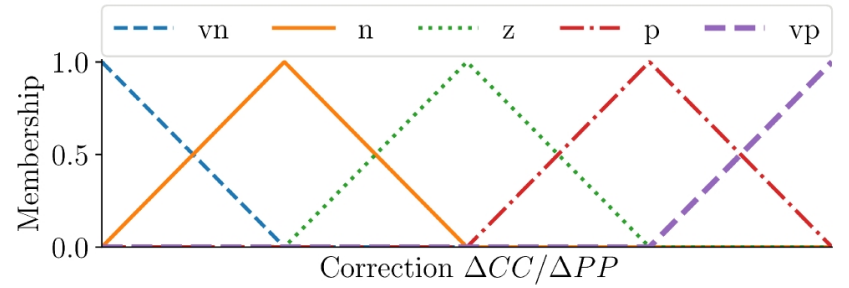

Fig. 2. Membership functions for corrections of cloud cover $(\triangle C C)$ and precipitation prediction $(\triangle P P)$.

The rules for output cloud cover and precipitation prediction corrections are defined in the Table II.

TABLE II. FUZZY RULE SET.

\begin{tabular}{|c|c|c|c|}
\hline$\Delta H$ & $\Delta P$ & $\Delta C C$ & $\Delta P P$ \\
\hline negative & positive & $\mathrm{vp}$ & $\mathrm{vp}$ \\
\hline negative & neutral & $\mathrm{p}$ & $\mathrm{p}$ \\
\hline negative & negative & $\mathrm{z}$ & $\mathrm{z}$ \\
\hline neutral & positive & $\mathrm{p}$ & $\mathrm{p}$ \\
\hline neutral & neutral & $\mathrm{z}$ & $\mathrm{z}$ \\
\hline neutral & negative & $\mathrm{n}$ & $\mathrm{n}$ \\
\hline positive & positive & $\mathrm{z}$ & $\mathrm{z}$ \\
\hline positive & neutral & $\mathrm{n}$ & $\mathrm{n}$ \\
\hline positive & negative & $\mathrm{vn}$ & $\mathrm{vn}$ \\
\hline
\end{tabular}

Defuzzified values of cloud cover and precipitation prediction correction, $\triangle C C$ and $\triangle P P$, are applied to the forecasted values up to $N_{F}$ hours in advance as follows:

$$
\begin{aligned}
& \widetilde{C C}^{t+i}=C C^{t+i}+\Delta C C \frac{N_{F}-i}{N_{F}}, \\
& \widetilde{P P}^{t+i}=P P^{t+i}+\Delta P P \frac{N_{F}-i}{N_{F}},
\end{aligned}
$$

where $i \in\left[0, N_{F}-1\right]$. The values $\widetilde{C C}^{t}$ and $\widetilde{P P}^{t}$ are used in (26) to calculate the expected solar irradiance.

Since weather forecast is not ideal, additional correction is applied by using solar energy prediction error to correct future predictions as

$$
\hat{E}^{t}=\min \left\{(1+\alpha) \tilde{E}^{t}, E_{C S}^{t}\right\}
$$

where

$$
\alpha=\frac{E^{t-1}-\widetilde{E}^{t-1}}{\widetilde{E}^{t-1}} .
$$

Instead of limiting $\alpha$ as in [19], we use the cloudless sky solar energy to limit the predicted energy after correction. This way, even if overestimation occurs, it is bound.

\section{Energy HARVESTING Wireless SENSOR NODE MODEL}

In order to evaluate predictors in terms of energy neutrality, we have modeled an Energy Harvesting Wireless Sensor Node (EHWSN) in a simulated environment. The node model is presented in Fig. 3.
The solar panel harvests solar energy. It is described by area $A$ and efficiency $\eta_{\text {panel }}$. We used a simplified version of the model from [27] to calculate the energy output of a solar panel in time period $T$ as follows

$$
E_{\text {panel }}=\eta_{\text {panel }} \tilde{G} \times A \times T,
$$

where $\widetilde{G}$ is predicted irradiance obtained from (26).
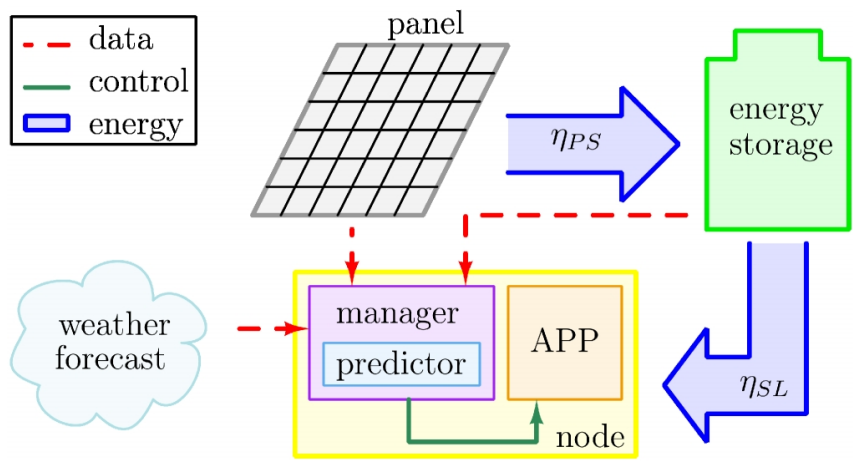

Fig. 3. Energy harvesting wireless sensor node model.

Harvested energy is stored in the energy storage with efficiency $\eta_{P S}$ because of energy conversions. The energy storage is modeled using state of charge $E_{E S}$ with maximum capacity of $E_{E S, M A X}$.

Sensor node consumes energy from storage with efficiency $\eta_{S N}$ because of conversions. It consumes average power of $P_{O N}$ when running at $100 \%$ duty cycle and $P_{O F F}$ when in idle mode. The maximum duty cycle is limited to $D C_{M A X}$, and average power that is consumed in that case is $P_{\text {MAX }}$.

Software node executes predefined application. The manager service is also present. It uses generated solar energy data from solar panel and current state of charge data from energy storage to adjust the node duty cycle. The manager also operates on weather forecast data obtained via the same wireless interface that is used to transmit measured data.

We have implemented a simple manager service for the evaluation of prediction algorithms in terms of energy neutrality. The service ensures that the state of charge information is used to adapt workload by adjusting the node duty cycle as shown in Fig. 4. The manager service implements only node-level energy management. However, implemented service is orthogonal to other node-level or network-level energy management algorithms.

As long as state of charge is above $E_{E S, H I}$, the node operates in regular mode and duty cycle is calculated based on current expected energy

$$
D C=\min \left\{\frac{\eta_{P S} \eta_{S N} \hat{E}^{t} / T-P_{O F F}}{P_{O N}-P_{O F F}}, D C_{M A X}\right\},
$$

where $T$ is time slot interval, which is 60 minutes. If predicted energy is enough to power the node with maximum duty cycle, then manager service selects 
maximum duty cycle $D C_{M A X}$.

If the state of charge falls below $E_{E S, H I}$, node operating mode is switched to conservative mode and duty cycle is set to $50 \%$ of the one obtained from (32) in order to allow the system to recover more quickly.

If the state of charge falls below $E_{E S, L O}$, the emergency mode is entered and duty cycle is reduced to $D C_{M I N}$. Once the state of charge rises again above $E_{E S, H I}$, the regular operating mode is entered and the duty cycle is again calculated based on the expected energy using (32).

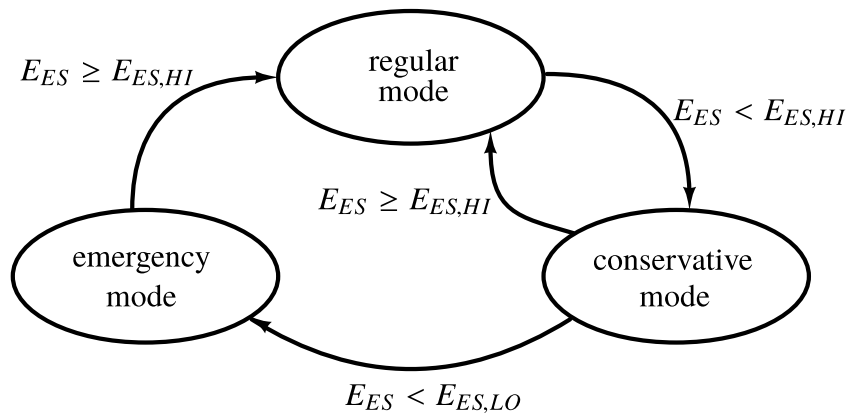

Fig. 4. FSM of manager service.

The expected energy for the following time slot $\hat{E}^{t}$ is used to set the duty cycle for the following time slot in the regular operating mode. If expected energy is above energy required to run at $D C_{L O}$ duty cycle

$$
E_{L O}=\left(D C_{L O} P_{O N}+\left(1-D C_{L O}\right) P_{O F F}\right) \times T
$$

the amount of energy that will be harvested is used to set the duty cycle as in (32), so the battery state of charge remains intact. The $D C_{L O}$ duty cycle is selected, so that energy storage starting at maximum capacity can sustain node operation for 12 hours without any harvested energy. In that case, the average power consumption in each time slot should be

$$
P_{a v g, L O}=\frac{1}{T} \frac{E_{E S, M A X}-E_{E S, H I}}{12},
$$

and $D C_{L O}$ can be calculated as

$$
D C_{L O}=\frac{P_{a v g, L O}-P_{O F F}}{P_{O N}-P_{O F F}} .
$$

If expected energy is below $E_{L O}$, as would happen at sunset, the further time slots are traversed until a time slot $t+X$ with $\hat{E}^{t+X}>E_{L O}$ is found, which is identified as sunrise. For time slots from $t$ to $t+X$, i.e., during the night, the average duty cycle is calculated and applied in the following manner

$$
D C_{\text {night }}=\frac{\eta_{S N}\left(\eta_{P S} \sum_{i=0}^{X-1} \hat{E}^{t+i}+E_{E S}^{t}-E_{E S, H I}\right)}{X \times T} .
$$

This way, energy storage state of charge at sunrise should be $E_{E S, H I}$ and node should continue to operate in regular mode. In addition, the energy storage state of charge between $E_{E S, L O}$ and $E_{E S, H I}$ is left as a margin, in case prediction errors occur.

\section{RESUlTS AND DISCUSSION}

Proposed predictor was tested in a simulated environment. Solar radiation data were obtained from Košutnjak automatic weather station [28] for a period from May to October 2018. Weather forecast data were obtained from Dark Sky [29], which offers application programming interface (API) access and data organized in JavaScript Object Notation (JSON) format.

Proposed predictor was compared to Pro-Energy [11], IPro-Energy [12], and our previous work [19] in terms of prediction error, as well on achieving energy neutrality.

Metrics used for calculation of prediction error are Mean Absolute Prediction Error (MAPE) (37), Mean Absolute Deviation Percent (MADP) (38), Normalized Root Mean Squared Error (nRMSE) (39), and Mean Bias Error (MBE) (40):

$$
\begin{gathered}
M A P E=\frac{100}{N} \sum_{i}\left|\frac{E^{i}-\hat{E}^{i} \mid}{E^{i}}\right| \\
M A D P=100 \times \frac{\sum_{M(i)}\left|E^{M(i)}-\hat{E}^{M(i)}\right|}{\sum_{M(i)} E^{M(i)}}, \\
n R M S E=100 \times \frac{\sqrt{\frac{1}{N} \sum_{i}\left(E^{i}-\hat{E}^{i}\right)^{2}}}{\frac{\sum_{i} E^{i}}{N}}, \\
M B E=\frac{1}{N} \sum_{i}\left(\hat{E}^{t}-E^{t}\right) .
\end{gathered}
$$

The MAPE metric is often used for evaluation of predictor quality. However, the downside of the MAPE metric is that when measured energy is close to zero, the error can be very high, which can skew the actual results. The MADP is used to calculate error across interval $M$ of consecutive time slots. Thus, MADP is more robust than MAPE, since it is not affected by low values of harvested energy. The normalized variant of RMSE is used in order to enable comparison with predictors tested on other data. The MBE is the only metric, which takes into account the sign of the prediction error, so it can show the overall trend of the prediction errors in terms of overestimation or underestimation.

Prediction errors have been calculated for the following time slot prediction. The interval $M$ for MADP calculation is set to 24 hours.

Modeled EHWSN parameters are selected according to the Table III. The CC430 WSN that was modeled in [30] is used as the sensor node.

Parameters of predictors that are used for comparison are selected according to appropriate papers: [11], [12], and [19]. The predictor from our previous paper [19] has no configurable parameters. The list of selected parameters is shown in Table IV. 
The fuzzy logic filter membership functions were configured based on the available data. The standard deviation of prediction errors for humidity and atmospheric pressure are $\sigma_{H}=11 \%$ and $\sigma_{P}=0.8 \mathrm{mbar}$, respectively. For $\Delta H_{1}, \Delta H_{2}, \Delta P_{1}$, and $\Delta P_{2}$ values, we chose the $3 \sigma$ range. The range of $\triangle C C$ is limited to $[-0.5,0.5]$ and $\triangle P P$ to $[-0.25,0.25]$.

Prediction errors in percent for all predictors are given in Table V.

TABLE III. SIMULATED EHWSN PARAMETER VALUES.

\begin{tabular}{|c|c|}
\hline Parameter & Value \\
\hline$A$ & $0.0033 \mathrm{~m}^{2}$ \\
\hline$\eta_{\text {panel }}$ & $15 \%$ \\
\hline$\eta_{P S}$ & $85 \%$ \\
\hline$E_{E S, M A X}$ & $932 \mathrm{~J}$ \\
\hline$\eta_{S N}$ & $95 \%$ \\
\hline$P_{O N}$ & $60 \mathrm{~mW}$ \\
\hline$P_{O F F}$ & $5 \mu \mathrm{W}$ \\
\hline$D C_{M A X}$ & $70 \%$ \\
\hline$D C_{M I N}$ & $0.01 \%$ \\
\hline$E_{E S, H I}$ & $0.2 \mathrm{EES}, \mathrm{MAX}$ \\
\hline$E_{E S, L O}$ & $0.05 \mathrm{EES}, \mathrm{MAX}$ \\
\hline
\end{tabular}

TABLE IV. SELECTED PARAMETER VALUES FOR EVALUATION OF PREDICTORS.

\begin{tabular}{|c|c|c|}
\hline Predictor & Parameter & Value \\
\hline \multirow{4}{*}{ Pro-Energy [11] } & $D$ & 14 \\
\hline & $K$ & 2 \\
\hline & $\alpha$ & 0.5 \\
\hline & $P$ & 9 \\
\hline \multirow{5}{*}{ IPro-Energy [12] } & $D$ & 30 \\
\hline & $K$ & 2 \\
\hline & $W_{f}$ & 0.7 \\
\hline & $P$ & 2 \\
\hline & $r$ & 0.5 \\
\hline previous [19] & \multicolumn{2}{|c|}{ no configurable parameters } \\
\hline \multirow{5}{*}{ presented } & $N_{f}$ & 6 \\
\hline & $\Delta H_{I}$ & -33 \\
\hline & $\Delta H_{2}$ & 33 \\
\hline & $\Delta P_{1}$ & -2.4 \\
\hline & $\Delta P_{2}$ & 2.4 \\
\hline
\end{tabular}

TABLE V. PREDICTION ERRORS OF EVALUATED PREDICTORS.

\begin{tabular}{|c|c|c|c|c|}
\hline Metric & $\begin{array}{c}\text { Pro-Energy } \\
{[\mathbf{1 1}]}\end{array}$ & $\begin{array}{c}\text { IPro-Energy } \\
{[\mathbf{1 2}]}\end{array}$ & $\begin{array}{c}\text { Previous } \\
{[\mathbf{1 9 ]}}\end{array}$ & Presented \\
\hline MAPE & 34.76 & 23.72 & 30.02 & 21.78 \\
\hline MADP & 14.14 & 13.40 & 17.07 & 4.75 \\
\hline nRMSE & 45.68 & 45.40 & 47.91 & 44.70 \\
\hline MBE & -17.90 & -30.69 & -31.30 & -9.63 \\
\hline
\end{tabular}

From Table V, the proposed predictor has the least prediction error for the following time slot looking at all four metrics. It achieves $8.2 \%, 27.4 \%$, and $37.3 \%$ less MAPE error than IPro-Energy, predictor presented in our previous paper [19], and Pro-Energy, respectively. Looking at MADP metric, proposed predictor has $64 \%$ less prediction error compared to second best predictor (IPro-Energy). The nRMSE errors are similar for all predictors with proposed predictor having 2-6\% less error. The MBE metric indicates that the proposed predictor has the least underestimate.

In Table VI, the average node duty cycle $\overline{D C}$ and total amount of time spent in emergency mode $\sum t_{e m}$ are shown.

Looking at the Table VI, the proposed predictor is the only predictor that does not enter the emergency mode. Proposed predictor enables $2 \%$ less average duty cycle than the predictor presented in our previous paper [19], but it does not enter emergency mode.

TABLE VI. NODE PERFORMANCE EVALUATION WHEN USING DIFFERENT PREDICTORS.

\begin{tabular}{|c|c|c|c|c|}
\hline Metric & $\begin{array}{c}\text { Pro-Energy } \\
{[\mathbf{1 1}]}\end{array}$ & $\begin{array}{c}\text { IPro-Energy } \\
{[\mathbf{1 2}]}\end{array}$ & $\begin{array}{c}\text { Previous } \\
{[\mathbf{1 9}]}\end{array}$ & Presented \\
\hline$\overline{D C}[\%]$ & 46.10 & 44.21 & 46.60 & 45.77 \\
\hline$\Sigma t_{e m}[\mathrm{~h}]$ & 39.33 & 3.63 & 42.50 & 0 \\
\hline
\end{tabular}

The distribution of MAPE prediction errors across different error ranges for evaluated predictors is shown in Table VII.

TABLE VII. PREDICTION ERROR DISTRIBUTION

\begin{tabular}{|c|c|c|c|c|}
\hline $\begin{array}{c}\text { Error } \\
\text { range }\end{array}$ & $\begin{array}{c}\text { Pro-Energy } \\
{[\mathbf{1 1}]}\end{array}$ & $\begin{array}{c}\text { IPro-Energy } \\
{[\mathbf{1 2}]}\end{array}$ & $\begin{array}{c}\text { Previous } \\
{[\mathbf{1 9 ]}}\end{array}$ & Presented \\
\hline $0.0-0.1$ & 58.80 & 60.46 & 60.69 & 65.43 \\
\hline $0.1-0.2$ & 9.85 & 8.45 & 8.33 & 8.85 \\
\hline $0.2-0.3$ & 6.82 & 7.68 & 6.89 & 6.00 \\
\hline $0.3-0.4$ & 5.51 & 3.80 & 5.23 & 4.76 \\
\hline $0.4-0.5$ & 4.27 & 3.52 & 4.62 & 3.43 \\
\hline $0.5-0.6$ & 3.15 & 3.34 & 2.92 & 2.50 \\
\hline $0.6-0.7$ & 1.89 & 2.82 & 1.38 & 1.49 \\
\hline $0.7-0.8$ & 1.10 & 2.26 & 1.05 & 1.31 \\
\hline $0.8-0.9$ & 0.75 & 2.05 & 0.89 & 0.68 \\
\hline $0.9-1.0$ & 0.79 & 0.65 & 0.49 & 0.65 \\
\hline$>1.0$ & 7.07 & 4.95 & 7.50 & 4.90 \\
\hline
\end{tabular}

From Table VII, proposed predictor has $74.28 \%$ of errors within the $20 \%$ MAPE range compared to $69 \%$ for other predictors. Also, looking at the last error range, where the percentage of MAPE errors is shown higher than $100 \%$, the proposed predictor has the lowest percentage of errors with only $4.90 \%$.

In order to get better insight into performance of the presented predictor, the MAPE error for sunlight hours from 05:00 to 19:00 is shown in Fig. 5.

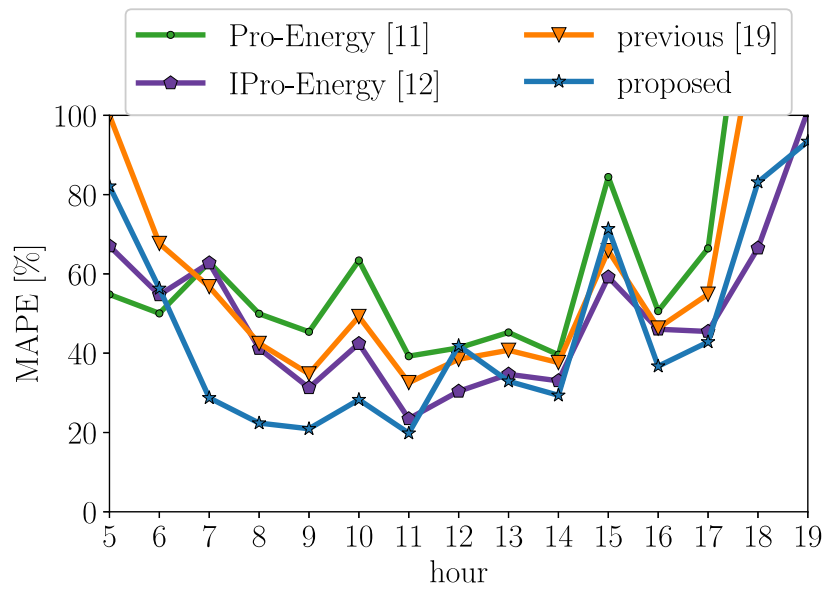

Fig. 5. MAPE errors across sunlight hours. 

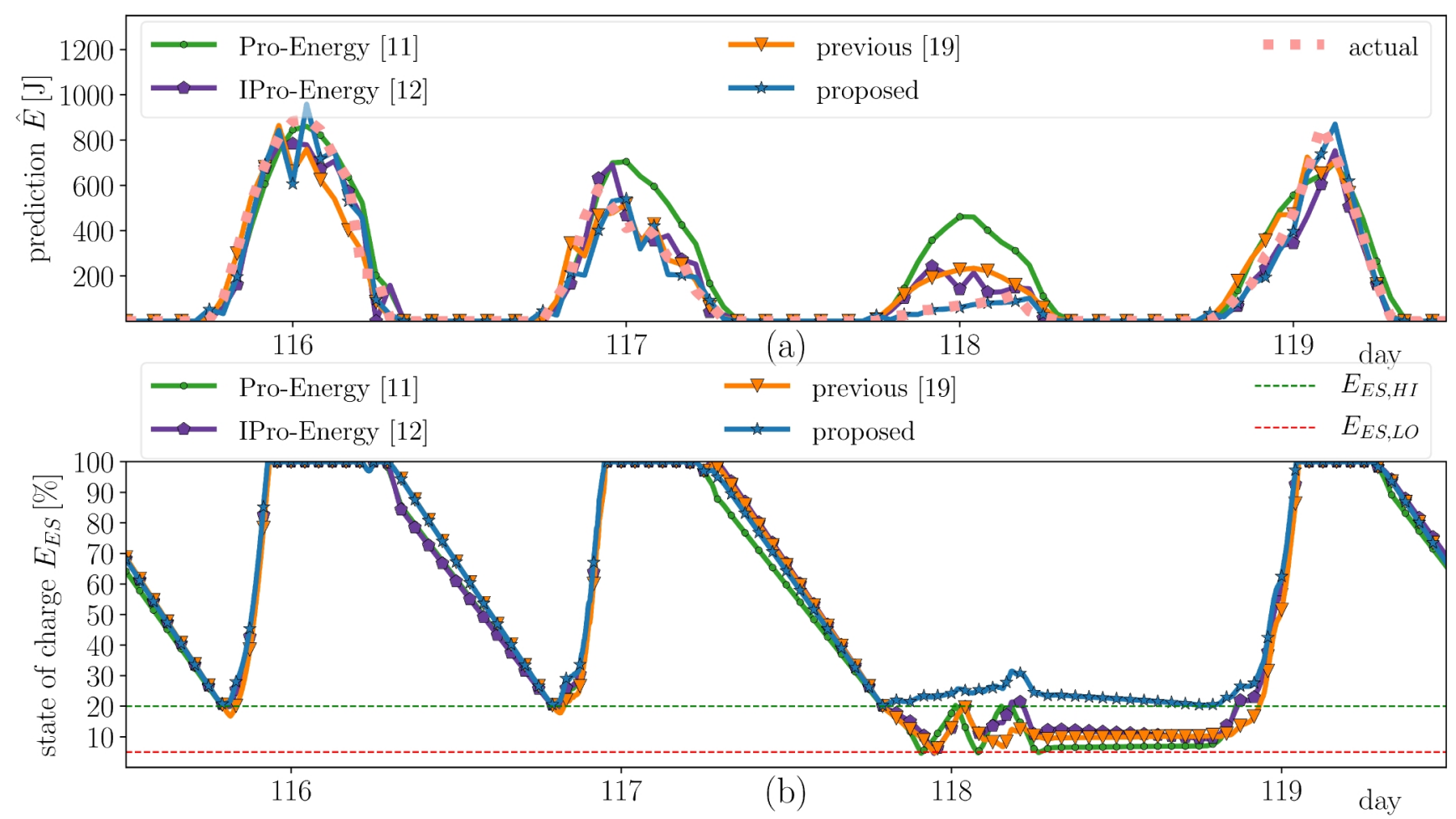

Fig. 6. Performance of evaluated predictors: a) Predicted harvested energy on days 116-119; b) Energy storage state of charge on days 116-119.

From Fig. 5, the presented predictor has lower MAPE error in the morning hours and maintains very good behaviour throughout the day.

The predicted harvested energy and energy storage state of charge for different predictors for four days of data are shown in Fig. 6. Figure 6(a) indicates that proposed predictor can track the measured data in a very good manner. In Fig. 6(b), it can be seen that the proposed predictor maintains battery state of charge around $E_{E S, H I}$, while other predictors can cause the state of charge to drop below $E_{E S, L O}$.

\section{CONCLUSIONS}

In this paper, we have presented an algorithm for harvested energy prediction for energy harvesting wireless sensor nodes. The predictor uses weather forecast data and applies corrections using fuzzy logic filter stage and correction based on prediction error. Since proposed predictor is targeted for application in wireless sensor nodes, the weather forecast information is obtained only once per day, at high noon, in order to minimize overhead. By using clear-sky radiation model, accurate information about maximum of solar radiation for each point in time can be calculated. Fuzzy logic filter enables correction of forecast results, which is especially useful when weather changes abruptly before next weather forecast data is obtained. The weather forecast data provides adequate long-term prediction capabilities, while applied corrections reduce the short-term prediction error. The presented predictor is configurable and can be integrated into different energy management schemes.

Presented predictor has been tested in a simulated environment using real life measurements and provides at least $8.2 \%$ less MAPE, $64 \%$ less MADP, and $2 \%$ less nRMSE prediction error compared to state-of-the-art predictors while also demonstrating the least prediction underestimate. Proposed predictor has the least MAPE in the morning hours, which is important, since prediction errors in the morning can lead to battery depletion.

Simulation results with the simple energy management algorithm show that the proposed predictor can provide energy neutral operation. Real-life experiments are planned for the future work.

\section{CONFLICTS OF INTEREST}

The authors declare that they have no conflicts of interest.

\section{REFERENCES}

[1] I. F. Akyildiz, W. Su, Y. Sankarasubramaniam, and E. Cayirci, "Wireless sensor networks: A survey", Computer Networks, vol. 38, pp. 393-422, Mar. 2002. DOI: 10.1016/S1389-1286(01)00302-4.

[2] T. Arampatzis, J. Lygeros, and S. Manesis, "A survey of applications of wireless sensors and wireless sensor networks", in Proc. of the 2005 IEEE International Symposium on, Mediterrean Conference on Control and Automation Intelligent Control, Limassol, 2005, pp. 719-724. DOI: 10.1109/.2005.1467103.

[3] S. Mansour, N. Nasser, L. Karim, and A. Ali, "Wireless sensor network-based air quality monitoring system", in Proc. of 2014 International Conference on Computing, Networking and Communications (ICNC), Honolulu, HI, 2014, pp. 545-550. DOI: 10.1109/ICCNC.2014.6785394.

[4] R. Silva, J. Sa Silva, and F. Boavida, "Mobility in wireless sensor networks - Survey and proposal", Computer Communications, vol. 52, pp. 1-20, Oct. 2014. DOI: 10.1016/j.comcom.2014.05.008.

[5] L. Atzori, A. Iera, and G. Morabito, "The internet of things: A survey", Computer Networks, vol. 54, pp. 2787-2805, Oct. 2010. DOI: $10.1016 /$ j.comnet.2010.05.010.

[6] L. G. Rios and J. A. I. Diguez, "Big data infrastructure for analyzing data generated by wireless sensor networks", in Proc. of 2014 IEEE International Congress on Big Data, Anchorage, AK, 2014, pp. 816 823. DOI: 10.1109/BigData.Congress.2014.142. 
[7] G. Anastasi, M. Conti, M. Di Francesco, and A. Passarella, "Energy conservation in wireless sensor networks: A survey", Ad Hoc Networks, vol. 7, pp. 537-568, May 2009. DOI: 10.1016/j.adhoc.2008.06.003.

[8] S. Sudevalayam and P. Kulkarni, "Energy harvesting sensor nodes: Survey and implications", IEEE Communications Surveys Tutorials, vol. 13, no. 3, pp. 443-461, 2011. DOI: 10.1109/SURV.2011.060710.00094.

[9] A. Kansal, J. Hsu, S. Zahedi, and M. B. Srivastava, "Power management in energy harvesting sensor networks", ACM Trans. Embed. Comput. Syst., vol. 6, no. 4, article no. 32, Sep. 2007. DOI: $10.1145 / 1274858.1274870$.

[10] J. R. Piorno, C. Bergonzini, D. Atienza, and T. S. Rosing, "Prediction and management in energy harvested wireless sensor nodes", in Proc. of 2009 1st International Conference on Wireless Communication, Vehicular Technology, Information Theory and Aerospace Electronic Systems Technology, Aalborg, 2009, pp. 6-10. DOI: 10.1109/WIRELESSVITAE.2009.5172412.

[11] A. Cammarano, C. Petrioli, and D. Spenza, "Pro-energy: A novel energy prediction model for solar and wind energy-harvesting wireless sensor networks", in Proc. of 2012 IEEE 9th International Conference on Mobile Ad-Hoc and Sensor Systems (MASS 2012), Las Vegas, NV, 2012, pp. 75-83. DOI: 10.1109/MASS.2012.6502504.

[12] Muhammad, H. K. Qureshi, U. Saleem, M. Saleem, A. Pitsillides, and M. Lestas, "Harvested energy prediction schemes for wireless sensor networks: Performance evaluation and enhancements", Wireless Communications and Mobile Computing, vol. 2017, article no. 6928325, 2017. DOI: 10.1155/2017/6928325.

[13] C. Renner and V. Turau, "Adaptive energy-harvest profiling to enhance depletion-safe operation and efficient task scheduling", Sustainable Computing: Informatics and Systems, vol. 2, no. 1, pp. 43-56, Mar. 2012. DOI: 10.1016/j.suscom.2012.02.001.

[14] A. Cammarano, C. Petrioli, and D. Spenza, "Online energy harvesting prediction in environmentally powered wireless sensor networks", IEEE Sensors Journal, vol. 16, no. 17, pp. 6793-6804, Sep. 2016. DOI: $10.1109 /$ JSEN.2016.2587220.

[15] N. Sharma, J. Gummeson, D. Irwin, and P. Shenoy, "Cloudy computing: Leveraging weather forecasts in energy harvesting senso systems", in Proc. of 2010 7th Annual IEEE Communications Society Conference on Sensor, Mesh and Ad Hoc Communications and Networks (SECON), Boston, MA, 2010, pp. 1-9. DOI: 10.1109/SECON.2010.5508260.

[16] Y. Li, Z. Jia, and X. Li, "Task scheduling based on weather forecast in energy harvesting sensor systems", IEEE Sensors Journal, vol. 14 no. 11 pp. 3763-3765, Nov. 2014. DOI: 10.1109/JSEN.2014.2327221.

[17] Y. Li, J. Si, and S. Ma, "Using energy-aware scheduling weather forecast based harvesting for reconfigurable hardware", IEEE
Transactions on Sustainable Computing, vol. 4, no. 1, pp. 109-117, 2019. DOI: 10.1109/TSUSC.2018.2800717.

[18] Ch. F. Wooldridge and S. Hayman, "Cloud cover assessment grids for whole-sky images", International Journal of Lighting Research and Technology, vol. 26, no. 1, pp. 9-12, 1994. DOI: $10.1177 / 096032719402600102$.

[19] S. Janković, D. El Mezeni, and L. Saranovac, "Improving energy usage in energy harvesting wireless sensor nodes using weather forecast", Telfor Journal, vol. 10, no. 1, pp. 38-43, 2018. DOI: $10.5937 /$ telfor $1801038 \mathrm{~J}$

[20] T. Huld, R. Müller, and A. Gambardella, "A new solar radiation database for estimating PV performance in Europe and Africa", Solar Energy, vol. 86, pp. 1803-1815, Jun. 2012. DOI: 10.1016/j.solener.2012.03.006

[21] C. Furlan and A. P. de Oliveira, "Hourly diffuse solar radiation in the presence of clouds and other environmental parameters: The city of São Paulo", Working Paper Series, no. 17, pp. 1-15, 2008.

[22] J. A. Duffie and W. A. Beckman, Solar Engineering of Thermal Processes. Wiley, 2013, ch. 2. DOI: 10.1002/9781118671603.

[23] H. C. Hottel, "A simple model for estimating the transmittance of direct solar radiation through clear atmospheres", Solar Energy, vol. 18 , no. 2, pp. 129-134, 1976. DOI: 10.1016/0038-092X(76)90045-1.

[24] B. Y. H. Liu and R. C. Jordan, "The interrelationship and characteristic distribution of direct, diffuse and total solar radiation", Solar Energy, vol. 4, pp. 1-19, Jul. 1960. DOI: 10.1016/0038092X(60)90062-1.

[25] N. Sharma, P. Sharma, D. Irwin, and P. Shenoy, "Predicting solar generation from weather forecasts using machine learning", in Proc. of 2011 IEEE International Conference on Smart Grid Communications (SmartGridComm), Brussels, 2011, pp. 528-533. DOI: 10.1109/SmartGridComm.2011.6102379.

[26] J. Rodway, P. Musilek, E. Lozowski, M. Prauzek, and J. Heckenbergerova, "Pressure-based prediction of harvestable energy for powering environmental monitoring systems", in Proc. of 2015 IEEE 15th International Conference on Environment and Electrical Engineering (EEEIC), Rome, 2015, pp. 725-730. DOI: 10.1109/EEEIC.2015.7165254.

[27] C. Tao, D. Shanxu, and C. Changsong, "Forecasting power output for grid-connected photovoltaic power system without using solar radiation measurement", in Proc. of the $2^{\text {nd }}$ International Symposium on Power Electronics for Distributed Generation Systems, Hefei, 2010, pp. 773-777. DOI: 10.1109/PEDG.2010.5545754.

[28] Republic Hydrometeorogical Service of Serbia, Meteorogical station Beograd - Automatic weather station Košutnjak. [Online]. Available: http://hidmet.gov.rs/eng/osmotreni/kosutnjak.php

[29] Dark Sky, "Powered By Dark Sky". [Online]. Available: https://darksky.net/poweredby

[30] S. Jankovic and L. Saranovac, "High-level power modeling of CC430 SoC", in Proc. of IcETRAN-2017, Kladovo, Serbia, 2017, p. ELI3.2. 